\title{
Revisiting Generalized Nash Games and Variational Inequalities
}

\author{
Ankur A. Kulkarni* Uday V. Shanbhag
}

September 15, 2010

\begin{abstract}
Generalized Nash games represent an extension of Nash games in which strategy sets are coupled across players. The equilibrium conditions of such a game can be compactly stated as a quasivariational inequality (QVI), an extension of the variational inequality (VI). Harker [9] showed that under certain conditions on the maps defining the QVI, a solution to a related VI solves the QVI. This is a particularly important result, given that variational inequalities are generally far more tractable than quasi-variational inequalities. This paper investigates the applicability of Harker's result to the class of generalized Nash games where the strategy sets are linked through a shared or common constraint. The application of Harker's result to the QVI associated with such games proves difficult because the hypotheses, that require that a set with certain properties exist, can fail to hold even for simple shared-constraint games. We show these hypotheses are in fact impossible to satisfy in most settings. But we show that for a modified QVI, whose solution set equals that of the original QVI, the hypothesis of Harker's result always hold. This paves the way for applying this result to shared-constraint games, albeit in an indirect manner. This avenue allows us to recover as a special case, a result proved by Facchinei et al. [4], in which it is shown that a suitably defined variational inequality provides a solution to the QVI of a shared-constraint Nash game.
\end{abstract}

\section{Introduction}

Variational inequalities (VIs) provide an avenue for compactly articulating equilibrium conditions for continuous-strategy Nash games. When these games are generalized to allow for coupled strategy sets, the variational conditions of the resulting games, referred to as generalized Nash games, are given by a quasi-variational inequality (QVI). In [9], Harker examined this class of games and showed that if a set satisfying certain conditions exists, a solution to a VI defined over this set provides a solution to the QVI. Yet, as observed by Facchinei et al. [4], this result is difficult to apply to QVIs that arise from an important class of generalized Nash games, namely generalized Nash games shared constraints (cf. [14]) in which the strategy sets across players are coupled by a common or shared constraint. The root of this difficulty is that even for the simplest of shared constraints, a set satisfying Harker's conditions is extraordinarily hard to find.

Motivated by these observations, this paper revisits Harker's conditions in depth to qualify their applicability to shared constraint games. Specifically, when Harker's result is applied to QVIs arising from shared constraint games, we show that there is at most one set that satisfies Harker's conditions and has a nonempty interior: it is the set that defines the shared constraint. Furthermore, if this set satisfies Harker's conditions, then it has to be cartesian in nature; in other words, Harker's result cannot

${ }^{*}$ Kulkarni and Shanbhag are in the Department of Industrial and Enterprise Systems Engineering at the University of Illinois at Urbana-Champaign. They are contactable at akulkar3@illinois.edu and udaybag@illinois.edu, respectively and would like to acknowledge the support of NSF award CCF-0728863 
be directly applied to most nontrivial shared constraint games ${ }^{1}$. As a result, we are able to formally explain why it remains a challenge to find a set satisfying Harker's conditions.

While it appears that Harker's result has reduced utility in the context of shared-constraint games, we observe that its applicability to nontrivial shared constraint games can be salvaged through an application to a 'modified' QVI. Specifically, we construct a modified QVI that admits the same solution set as the original QVI, and for this modified QVI, there always exists a set satisfying Harker's conditions. In short, Harker's result applies to all nontrivial shared constraint games when it is applied through the modified QVI, though it fails when applied directly. As a corollary we also see that Harker's result implies a result provided by Facchinei et al. [4] as an alternative to Harker's result.

While QVIs are natural tools for modeling game-theoretic problems, these objects are analytically harder to handle than VIs. Therefore, any results that reduce the analysis to a VI are particularly important and those provided by Harker and Facchinei et al. are, to the best of our knowledge, the only ones that enable such a reduction. We believe that our work clarifies their reach, revealing a surprising relationship between these two results, and allowing for the application of Harker's result through the modified QVI.

In Section 2, we introduce generalized Nash games with shared constraints and the associated QVI and VI. In Section 3, we examine Harker's conditions in depth and clarifies their shortcomings. Section 4 discusses the how application of Harker's result to a modified QVI avoids these shortcomings. Additionally, we show how Facchinei et al.'s result may be obtained through this application. Some concluding remarks are provided in Section 5.

\section{Preliminaries}

Let $\mathcal{N}=\{1,2, \ldots, N\}$ be a set of players, $m_{1}, \ldots, m_{N}$ be positive integers and $m=\sum_{i=1}^{N} m_{i}$. For each $i \in \mathcal{N}$, let $x_{i} \in \mathbb{R}^{m_{i}}$ be his strategy and $\varphi_{i}: \mathbb{R}^{m} \rightarrow \mathbb{R}$ be his objective function. We use the following notation: by $x$, we denote the tuple $\left(x_{1}, x_{2}, \ldots, x_{N}\right), x^{-i}$ denotes the tuple $\left(x_{1}, \ldots, x_{i-1}, x_{i+1}, \ldots, x_{N}\right)$ and $\left(y_{i}, x^{-i}\right)$ the tuple $\left(x_{1}, \ldots, x_{i-1}, y_{i}, x_{i+1}, \ldots, x_{N}\right)$. int $(\bullet)$ and $\partial \bullet$ denote the interior and boundary of ' $\bullet$ ' respectively. A shared constraint is a requirement that the tuple $x$ be constrained to lie in a set $\mathbb{C} \subseteq \mathbb{R}^{m}$. In the generalized Nash game with shared constraint $\mathbb{C}$, player $i$ is assumed to solve the parameterized optimization problem

\begin{tabular}{|lll|}
\hline $\mathrm{A}_{i}\left(x^{-i}\right)$ & $\begin{array}{l}\operatorname{minimize}_{x_{i}} \\
\text { subject to }\end{array}$ & $x_{i}\left(x_{i} ; x^{-i}\right)$ \\
&
\end{tabular}

where for each $i \in \mathcal{N}$, the set-valued maps $K_{i}: \prod_{j \neq i} \mathbb{R}^{m_{j}} \rightarrow 2^{\mathbb{R}^{m_{i}}}$ and the map $K: \mathbb{R}^{m} \rightarrow 2^{\mathbb{R}^{m}}$, are defined as

$$
K_{i}\left(x^{-i}\right):=\left\{y_{i} \in \mathbb{R}^{m_{i}} \mid\left(y_{i}, x^{-i}\right) \in \mathbb{C}\right\}, \quad \forall i \in \mathcal{N} \text { and } \quad K(x):=\prod_{i \in \mathcal{N}} K_{i}\left(x^{-i}\right) \quad \forall x \in \mathbb{R}^{m} .
$$

Definition 1 (Generalized Nash equilibrium (GNE)) A strategy tuple $x \equiv\left(x_{1}, x_{2}, \ldots, x_{N}\right)$ is a generalized Nash equilibrium of this game if $x_{i} \in S O L\left(A_{i}\left(x^{-i}\right)\right)$ for all $i \in \mathcal{N}$.

Arrow and Debreu [1] were the first to consider games ${ }^{2}$ where dependence on opposition strategies was allowed in the constraints. Rosen [14] was the first to study the particular setting where the players'

\footnotetext{
${ }^{1}$ Nontrivial shared constraint games allude to games where the set defining the shared constraint is not cartesian or rectangular.

${ }^{2}$ Arrow and Debreu termed these games as "abstract economies".
} 
strategies were coupled through a common or shared constraint. Shared constraint games have a host of applications and are a subject of active research (see e.g., [5]).

A quasi-variational inequality is defined by a set-valued map $L$ and a function $G$ and is the following problem.

$$
\text { Find } x \in L(x) \quad \text { such that } \quad G(x)^{T}(y-x) \geq 0 \quad \forall y \in L(x) .
$$

For any closed convex set $U \subseteq \mathbb{R}^{m}$ and function $G: U \rightarrow \mathbb{R}^{m}$, the variational inequality $\operatorname{VI}(U, G)$ is the following problem.

$$
\text { Find } x \in U \quad \text { such that } \quad G(x)^{T}(y-x) \geq 0 \quad \forall y \in U \text {. }
$$

We make the following assumptions throughout the paper.

Assumption 2 For each $i \in \mathcal{N}$, the objective function $\varphi_{i} \in C^{1}$ and $\varphi_{i}\left(x_{i} ; x^{-i}\right)$ is convex in $x_{i}$ for all $x^{-i}$. Unless otherwise mentioned, $\mathbb{C}$ is closed and convex set.

A vector $x=\left(x_{1}, \ldots, x_{N}\right)$ is a GNE of the above game if and only if it solves the quasi-variational inequality $\mathrm{QVI}(K, F)[6]$, where

$$
F(x)=\left(\begin{array}{c}
\nabla_{1} \varphi_{1}(x) \\
\vdots \\
\nabla_{N} \varphi_{N}(x)
\end{array}\right),
$$

and $\nabla=\frac{\partial}{\partial x_{i}}$. Critical to all our theorems is the nature of the set-valued map $K$. This has been investigated in depth in [11]. Some properties of $K$ that will be useful in the results that follow are listed below. The proofs for these claims can be found in [11]. They have also been reproduced in the Appendix.

Lemma 3 Let $\mathbb{C}$ be a closed set in $\mathbb{R}^{m}$ and $K$ be as given in (1). Then the following hold:

(a) If $\mathbb{C}=\prod_{i \in \mathcal{N}} \mathbb{C}_{i}$, where $\mathbb{C}_{i} \subseteq \mathbb{R}^{m_{i}}$ for every $i \in \mathcal{N}$, are nonempty, not necessarily convex sets, then $K(x)=\mathbb{C}$ for every $x$ in $\mathbb{C}$ and is empty otherwise.

(b) For any $\mathbb{C}$, not necessarily convex, $x$ is a fixed point of $K$ if and only if $x \in \mathbb{C}$.

(c) If $\mathbb{C}$ is closed and convex, $K(x)$ is closed and convex for any $x \in \operatorname{dom}(K)$.

(d) A point $x$ belongs to the interior of $K(x)$ if and only if $x$ is in the interior of $\mathbb{C}$.

\section{Harker's conditions}

We may better understand Harker's result by seeing it within the history of results on QVIs. The QVI was first introduced by Bensoussan, Goursat and Lions [2] and is a significantly harder problem than the VI. Most of the challenges in the analysis of the QVI can be traced to the set-valued map in its definition. Therefore, many of first existence results on QVIs rested on the well-behavedness of this map, as evidenced, for e.g., by the results of Ichiishi [10] and Chan and Pang [3] which rely on the continuity of the set-valued map and compactness of its graph. Unfortunately, continuity is too stringent a requirement that is seen to fail for many simple settings. In [9], Harker gave an important result that circumvents the need for continuity by constructing a VI whose solutions solve the QVI. 


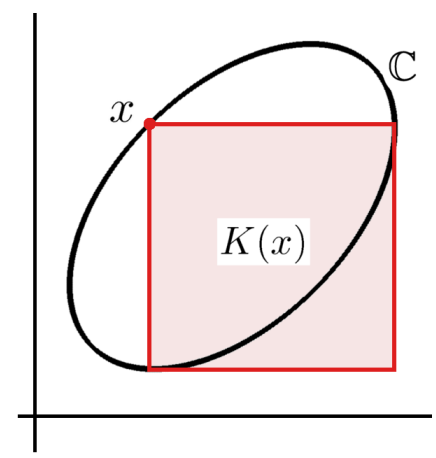

Figure 1: A set $\mathbb{C}, x$ on the boundary of $\mathbb{C}$ and $K(x)$.

Since numerous existence results were known for VIs, these could potentially be leveraged to claim the existence of a solution to the QVI without invoking the continuity of the set-valued map. We begin our discussion by reproducing ${ }^{3}$ Theorem 3 from Harker's paper [9].

Theorem 4 (Harker [9]) Let $F$ and $L$ be respectively point-to-point and point-to-set mappings from $\mathbb{R}^{m}$ to itself. Suppose that there exists a nonempty closed, convex set $A$ such that

(i) $L(x) \subseteq A$ for all $x \in A$ and

(ii) $x \in L(x)$ for all $x \in A$.

Then any solution to the variational inequality $V I(A, F)$ is a solution of $Q V I(L, F)$

It is easy to see what Harker is attempting to do: in (ii) he seeks that all points in the set $A$ are feasible for the QVI and in (i) he requires that $A$ be large enough to subsume the moving set $L(x)$ for each $x$ in $A$. The continuity of $L$ does not appear in the picture.

Theorem 4 is not limited to QVIs arising from generalized Nash games with shared constraints. i.e. $L$, in Theorem 4, is not required to be of the form specified in (1) for $K$. However, problems emerge when one tries to apply it with $L=K$. Consider the following: any set $A$ satisfying (ii) must be a subset of $\mathbb{C}$. But combining this with (i) implies that $K(x)$ must be a subset of $\mathbb{C}$ for all $x \in A$. In the trivial setting where $\mathbb{C}$ is rectangular (or cartesian, cf. Lemma $3(\mathrm{a})$ ), $K(\cdot) \equiv \mathbb{C}$ and (i) and (ii) obviously hold. But when $\mathbb{C}$ is not rectangular, $K(x)$ appears to routinely include points outside $\mathbb{C}$ for any $x$ in $\mathbb{C}$. For e.g., in Fig. 1, for a given $x \in \mathbb{C}$, one of the corners of $K(x)$ lies outside $\mathbb{C}$. Even in the simple case where $\mathbb{C}$ is polyhedral, $K$ does not stay confined within $\mathbb{C}$. This was also observed by Facchinei et al. [4] through the following example.

Example 1. (Facchinei et al. [4]) Suppose $\mathbb{C}$ is defined as

$$
\mathbb{C} \triangleq\left\{\left(x_{1}, x_{2}\right) \in \mathbb{R}^{2} \mid x_{1}+x_{2} \leq 2, x_{2}-x_{1} \geq 0, x_{1}, x_{2} \geq 0\right\} .
$$

Then $\mathbb{C}$ is a triangle in $\mathbb{R}^{2}$ with vertices $(0,0),(1,1)$ and $(0,2)$. Let $A=\mathbb{C}$ and consider $(0,1) \in \mathbb{C}$. Then, we have

$$
K(0,1)=[0,1] \times[0,2] .
$$

\footnotetext{
${ }^{3}$ Theorem 4 is a slight modification Harker's Theorem 3 in [9]. Harker's version also requires that the set ' $A$ ' in Theorem 4 to be compact, whereas we have required only closedness on $A$. Compactness is imposed presumably in keeping with the assumption prevailing in [9] that each player has compact strategy sets. It is trivial to check that the result is valid even under the closedness $A$.
} 
Clearly, $K(0,1)$ is a rectangle that includes in it the triangle $\mathbb{C}$, implying that Theorem 4 (i) is not satisfied.

Consequently, when $L=K$, finding a set $A$ satisfying (i) and (ii) proves extraordinarily difficult and one is led to the question of whether there exists any nontrivial $\mathbb{C}$ for which Theorem 4 can be applied with this $L$.

The goal of this section is to show that if one also requires $A$ to have a interior, the trivial setting where $\mathbb{C}$ is rectangular is indeed the only setting where Theorem 4 applies with $L=K$. We show this through a combination of two results, the first of which is Theorem 5 . Theorem 5 says that when $L=K$, there is at most one closed convex set $A$ that satisfies (i), (ii) and has a nonempty interior and this set has to be $\mathbb{C}$ itself. The second result is Theorem 6 which says that if $\mathbb{C}$ has a nonempty interior and satisfies (i), (ii) with $L=K$, then $\mathbb{C}$ has to be rectangular.

Theorem 5 Let $\mathbb{C} \subseteq \mathbb{R}^{m}$ be closed and convex with nonempty interior and $K$ be as defined in (1). Let $A \subseteq \mathbb{R}^{m}$ and consider the following three conditions:

(a) A is closed, convex, and has nonempty interior

(b) $K(x) \subseteq A$ for all $x \in A$

(c) $x \in K(x)$ for all $x \in A$.

If $A$ satisfies (a), (b), and (c), then $A=\mathbb{C}$. Therefore,

1. If $\mathbb{C}$ satisfies the above three conditions, it is the only set satisfying these conditions.

2. If $\mathbb{C}$ does not satisfy these conditions, there does not exist any set that satisfies them.

Proof : Let $A$ satisfy conditions (a), (b), (c). If $A=\mathbb{C}$, it is easy to see that claims (1) and (2) follow logically. So we will prove $A=\mathbb{C}$.

(c) says that every point in $A$ is a fixed point of $K$. So by Lemma 3 (b) we must have $A \subseteq \mathbb{C}$. It follows that $\operatorname{int}(A) \subseteq \operatorname{int}(\mathbb{C})$. We will first show that $\partial A \cap \operatorname{int}(\mathbb{C})=\emptyset$ and use that to conclude $A=\mathbb{C}$. If $\partial A \cap \operatorname{int}(\mathbb{C}) \neq \emptyset$, we can pick any $x \in \operatorname{int}(\mathbb{C}) \cap \partial A$ and construct a ball $B$ around $x$ such that $B \subseteq \operatorname{int}(\mathbb{C}) \cap \operatorname{int}(K(x))$ (this is ensured by Lemma 3(d)). But using (b), we see that $B$, which is a subset of $K(x)$, has to be included in $A$. Since a ball around $x$ is included in $A$, this means that $x$ is in the interior of $A$. But this contradicts the assumption that $x$ lies on the boundary of $A$. So $\partial A \cap \operatorname{int}(\mathbb{C})$ must be empty and that $\partial A$ must be included in $\partial \mathbb{C}$.

So we have $A \subseteq \mathbb{C}$ with $\operatorname{int}(A) \subseteq \operatorname{int}(\mathbb{C})$ and $\partial A \subseteq \partial \mathbb{C}$. We now show that this implies $A=\mathbb{C}$. The argument goes via contradiction. Suppose that there is a point $y \in \mathbb{C}$ which is not in $A$. Take some other point $z \in \operatorname{int}(A) \subseteq \operatorname{int}(\mathbb{C})$ and consider the segment joining $y$ and $z$. It is known (see $[13$, Theorem 6.1]) that the points $\{t y+(1-t) z, t \in[0,1)\}$ lie in $\operatorname{int}(\mathbb{C})$. If we can show that the segment $\{t y+(1-t) z \mid t \in(0,1)\}$ crosses the boundary ${ }^{4}$ of $A$, it will contradict $\partial A \cap \operatorname{int}(\mathbb{C})=\emptyset$ and complete the proof. Suppose the set $\{t y+(1-t) z \mid t \in(0,1)\}$ does not cross the boundary of $A$. Then the interval $(0,1)$ is the disjoint union of the following sets

$$
T_{0}=\{t \in(0,1) \mid t y+(1-t) z \in \operatorname{int}(A)\} \quad \text { and } \quad T_{1}=\left\{t \in(0,1) \mid t y+(1-t) z \in A^{c}\right\} .
$$

Recall that $z \in \operatorname{int}(A)$ and $y \in A^{c}$. Since $A$ is convex, $\operatorname{int}(A)$ is convex. So $T_{0}$ is a convex subset of $(0,1)$. Furthermore, $T_{0}$ is open at 0 and open at the other end. i.e. $T_{0}=(0, \alpha)$ for some $\alpha \in(0,1)$.

\footnotetext{
${ }^{4}$ While this may seem intuitively obvious, it is nontrivial. In the most general setting the result is called "Jordan separation theorem" [7]. We have given an argument based on completeness of reals, inspired by [8, page 38-40].
} 
Consequently $T_{1}=[\alpha, 1)$. But since $T_{1}$ is open at both ends, this is a contradiction. So the segment $\{t y+(1-t) z \mid t \in(0,1)\}$ intersects the boundary of $A$. This contradicts our conclusion that int $(\mathbb{C})$ and the $\partial A$ are disjoint. So our assumption that there exists a $y \in \mathbb{C} \cap A^{c}$ is incorrect, and we must indeed have $A=\mathbb{C}$.

Therefore, if we apply Theorem 4 with $L=K$ by also requiring that the set $A$ have a nonempty interior, then there is at most one choice for $A$ : $\mathbb{C}$ itself. Consequently, if $\mathbb{C}$ does not satisfy Theorem 4(i),(ii), it is not possible to find an $A$ with nonempty interior satisfying Theorem 4(i),(ii). Next, one may ask what kind of a set may $\mathbb{C}$ be so as to satisfy conditions (a), (b) and (c) in Theorem 5. The following theorem shows if $\mathbb{C}$ satisfies these conditions then $\mathbb{C}$ has to be rectangular. This is an important finding in that we can essentially claim that Harker's result cannot be applied to many non-trivial shared constraint games.

Theorem 6 Suppose $\mathbb{C} \subseteq \mathbb{R}^{m}$ and let $K$ be as defined in (1). If $A=\mathbb{C}$ satisfies (a), (b), (c) from Theorem 5, then there exist for each $i \in \mathcal{N}$, sets $\mathbb{C}_{i} \subseteq \mathbb{R}^{m_{i}}$ such that $\mathbb{C}=\prod_{i \in \mathcal{N}} \mathbb{C}_{i}$.

Proof : It suffices to show that int $(\mathbb{C})$ is rectangular. We show this through a series of steps that follow the following argument. The first step claims that the union $\bigcup_{x \in \operatorname{int}(\mathbb{C})} \operatorname{int}(K(x))$ is the set int $(\mathbb{C})$. Steps 2,3 together show that $K$ is locally constant on $\operatorname{int}(\mathbb{C})$. In Step 4 , sing the convexity of int $(\mathbb{C})$ we show that $K$ is in fact constant on $\operatorname{int}(\mathbb{C})$. Combining this with the first step gives the result.

Step 1. $\bigcup_{x \in \operatorname{int}(\mathbb{C})} \operatorname{int}(K(x))=\operatorname{int}(\mathbb{C})$.

Proof: From (b) in Theorem 5, $\operatorname{int}(K(x)) \subseteq \operatorname{int}(\mathbb{C})$ and thus $\bigcup_{x \in \operatorname{int}(\mathbb{C})} \operatorname{int}(K(x)) \subseteq \operatorname{int}(\mathbb{C})$. But from Lemma $3(\mathrm{~d})$, we get $\operatorname{int}(\mathbb{C}) \subseteq \bigcup_{x \in \operatorname{int}(\mathbb{C})} \operatorname{int}(K(x))$, whereby the claim follows.

Step 2. If $x, y \in \mathbb{C}$, then $y \in K(x) \Longleftrightarrow x \in K(y)$.

Proof: Let $y \in K(x)$. It follows that $y_{i} \in K_{i}\left(x^{-i}\right)$ and $x_{i} \in K_{i}\left(x^{-i}\right)$ for all $i$. Therefore for all $i$, $\left(x_{i}, y^{-i}\right) \in K(x)$. But Theorem 5 implies that if $\left(x_{i}, y^{-i}\right) \in K(x)$ then $\left(x_{i}, y^{-i}\right) \in \mathbb{C}$. Therefore by (1), $x \in K(y)$. Since $x$ and $y$ are arbitrary, the equivalence $y \in K(x) \Longleftrightarrow x \in K(y)$ follows.

Step 3. If $x, y \in \mathbb{C}$ and $y \in K(x)$, then $K(y)=K(x)$.

Proof: As a consequence of Step 2, we have that if $y \in K(x)$, then $x \in K(y)$. We first proceed to show that $K(x) \subseteq K(y)$. Let $z$ be a point in $K(x)$. For each $i,\left(z_{i}, y^{-i}\right) \in K(x)$. Arguing as in Step 2, (b) implies that $z \in K(y)$, whereby $K(x) \subseteq K(y)$. Proceeding in an identical fashion from $x \in K(y)$, we have that $K(y) \subseteq K(x)$. It follows that $K(y)=K(x)$.

$\underline{\text { Step } 4 .} K$ is constant on $\operatorname{int}(\mathbb{C})$.

Proof: We proceed to show this result by first showing that $K$ is constant over a line segment $[x, y]$ where $x, y \in \operatorname{int}(\mathbb{C})$. Since $\mathbb{C}$ is convex the segment joining $x$ and $y,[x, y]$ lies in the interior of $\mathbb{C}$. For any $z \in[x, y]$, let $B_{z}$ be an open ball contained in $\mathbb{C} \cap K(z)$ (cf Lemma 3(d)). It follows that

$$
\mathcal{V}:=\left\{B_{z} \mid z \in[x, y]\right\}
$$

is an open cover of $[x, y]$. Since $[x, y]$ is compact, there exists a finite subcollection, $\mathcal{U}$, of $\mathcal{V}$ such that

$$
[x, y] \subseteq \bigcup_{B \in \mathcal{U}} B
$$


We number balls in $\mathcal{U}$ inductively such that at any stage of numbering, $K$ is constant over the union of the numbered balls. Since $\mathcal{U}$ has finitely many balls, this process will end with $K$ being constant over $\mathcal{U}$. To begin, choose an arbitrary ball from $\mathcal{U}$ and call it $B_{1}$. By Step $3, K$ is constant on $B_{1}$ with value $K\left(z^{1}\right)$, where $z^{1}$ is such that $B_{z^{1}}=B_{1}$. Now suppose that $\ell$ balls have been numbered, where $1 \leq \ell \leq|\mathcal{U}|$ and $K$ is constant over $\cup_{j=1}^{\ell} B_{j}$ and let $\overline{\mathcal{U}}_{\ell}$ be the set of balls that are yet to be numbered. The unions $\cup_{j=1}^{\ell} B_{j}$ and $\cup_{B \in \overline{\mathcal{U}}_{\ell}} B$ are open sets. If these unions are disjoint, we would get that $[x, y]$ is disconnected, which is absurd, since $[x, y]$ is convex. Therefore there exists a ball $B_{\ell+1}$ where in $\overline{\mathcal{U}}_{\ell}$ such that $B_{\ell+1} \cap \cup_{j=1}^{\ell} B_{j} \neq \emptyset$. It follows that $K$ is constant over $B_{1}, \ldots, B_{\ell+1}$. Continuing in this manner we get $K$ is constant over $\cup_{B \in \mathcal{U}} B$, and in particular, over $[x, y]$. Since $x$ and $y$ were arbitrary points in $\operatorname{int}(\mathbb{C})$, we get $K$ is constant over $\operatorname{int}(\mathbb{C})$.

Step 5. $\operatorname{int}(\mathbb{C})$ is rectangular.

Proof: Since $K$ is constant over $\operatorname{int}(\mathbb{C})$, the conclusion of Step 1 degenerates to $\operatorname{int}(\mathbb{C})=\operatorname{int}(K(x))$ for each $x \in \operatorname{int}(\mathbb{C})$. But since $K$ is rectangular, $\operatorname{int}(\mathbb{C})$ is rectangular.

We may summarize the conclusions of Theorems 5 and 6 as follows. When $L=K$, Theorem 5 limits our choice for sets $A$ with nonempty interior that satisfy Harker's conditions to only the set $\mathbb{C}$. But Theorem 6 goes to show that $\mathbb{C}$ cannot be a choice for $A$ for verifying Harker's conditions with $L=K$ unless $\mathbb{C}$ is rectangular. But in this case, $\mathbb{C}$ isn't really a shared constraint at all and the $\mathrm{QVI}(K, F)$ is the same as $\operatorname{VI}(\mathbb{C}, F)$. It is therefore safe to conclude that taking $L=K$ is not the best way to apply Theorem 4 to shared constraint games. Section 4 presents an alternative way of applying Theorem 4 .

Some technical remarks are worth mentioning at this juncture regarding the above proof. First, it is well known (see, e.g., p. 114, [12]) that a locally constant function on a connected space is constant. Presumably, such a result also holds for set-valued maps and Step 4 could potentially be claimed through this result. Unfortunately, we are not aware of any such result.

Secondly, we note some caveats about Theorem 5 and Theorem 6 . That the set $A$ sought by Theorem 5 must have an interior is an important ingredient for the validity of Theorem 5 . The argument, that any line joining a point inside $A$ to a point outside $A$ must cross the boundary, used in proving Theorem 5 holds only when $A$ has an interior. In fact we have an example in [11] of an $A$ without an interior that satisfies Harker's conditions for a specific $\mathbb{C}$. The set $\mathbb{C}$ is such a point $x^{*} \in \partial \mathbb{C}$ exists, for which $K\left(x^{*}\right)$ is the singleton $\left\{x^{*}\right\}$. Clearly one may take $A=\left\{x^{*}\right\}$ to verify Harker's conditions. We have reproduced the relevant portions of the example here.

Example 2. Fig. 2 shows $\mathbb{C} \subseteq \mathbb{R}^{2}$ and a point $x^{*} \in \partial \mathbb{C}$ with the property that the image of $x^{*}$ under $K$ is a singleton, namely $x^{*}$ itself. In Fig. 2, dotted lines depict axes with their origin shifted to $x^{*}$. If $y \in K\left(x^{*}\right)$, the points $\left(y_{1}, x_{2}^{*}\right)$ and $\left(x_{1}^{*}, y_{2}\right)$ lie in on these 'axes'. Notice that since these 'axes' intersect $\mathbb{C}$ at only one point, $x^{*}, K\left(x^{*}\right)=\left\{x^{*}\right\}$.

The nonemptiness of $\operatorname{int}(\mathbb{C})$ is also essential to Theorem 6 , as seen in the following example.

Example 3. Let $\mathbb{C}=\left\{\left(x_{1}, x_{2}\right) \in \mathbb{R}^{2} \mid\left(x_{1}=x_{2}\right)\right\}$. Suppose $\mathbb{C}$ is a line in $\mathbb{R}^{2}$ and has no interior. For any $x \in \mathbb{C}$, we have $K(x)=\{x\}$. Clearly $A=\mathbb{C}$ satisfies (b) and (c) from Theorem 5 , but it does not satisfy (a).

\section{Applying Harker's conditions to the modified QVI}

Facchinei et al. have rightly observed in [4, section 4] that Harker's result is hard to use if $L=K$. In [4, p. 162], the authors state "The problem with this (Harker's) result is, we believe, that it is not simple to 


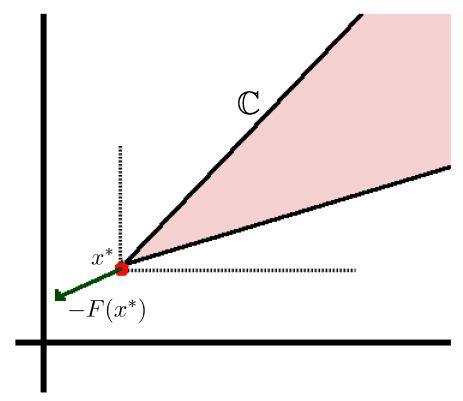

Figure 2: An example where $K\left(x^{*}\right)=\left\{x^{*}\right\}$.

give classes of problems for which (i) and (ii) are satisfied for some easily calculated A. Rosen's setting (i.e. generalized Nash games with shared constraints) analyzed in this paper is certainly not covered (when $A=\mathbb{C}$, as suggested in Harker's paper for polyhedral $\mathbb{C}$ ). In fact, in Rosen's setting, condition (ii) is obviously always satisfied, but condition (i) need not, except when $\mathbb{C}$ is a rectangle." and provide Example 1 to illustrate this difficulty.

As an alternative to Harker's result, Facchinei et al. gave a result that prescribed a VI associated with a shared-constraint game that provided a solution to the original QVI ([4, Theorem 2.1]). We present this result next.

Theorem 7 (Facchinei et al. [4]) Let $F$ be continuous and $K$ be as defined by (1). Then every solution of $V I(\mathbb{C}, F)$ is a solution of $Q V I(K, F)$.

Unlike Harker's result, this result is more direct and clearly applies to all shared constraint games.

It is evident from [4] and Example 1 that the observations made by Facchinei et al. were based on taking $L$ to be the map $K$ defined in (1). Indeed, our results from Section 3 formalize and substatiante their observations. But despite the results from Section 3, Harker's result is still of relevance to shared constraint games. Our contention is that the difficulty of applying Harker's result arises from taking $L=K$ and in assuming that this is the only way to apply Harker's result. Harker's result is better applied to a modified $\mathrm{QVI}(K \cap \mathbb{C}, F)$ as opposed to a direct application to $\mathrm{QVI}(K, F)$. When applied in this manner, Harker's result implies the Theorem 7 of Facchinei et al. We elaborate on this in this section. Consider $\mathrm{QVI}(K \cap \mathbb{C}, F)$ defined as

Find $x \in K(x) \cap \mathbb{C}$ such that $\quad F(x)^{T}(y-x) \geq 0 \quad \forall y \in K(x) \cap \mathbb{C}$. $(\mathrm{QVI}(K \cap \mathbb{C}, F))$

This seemingly weaker QVI is in fact equivalent to $\mathrm{QVI}(K, F)$.

Proposition 8 Let $F$ be continuous and $K$ be as defined by (1). Then, we have

$$
S O L(Q V I(K, F))=S O L(Q V I(K \cap \mathbb{C}, F)) .
$$

Proof : "ᄃ" If $x$ solves $\mathrm{QVI}(K, F)$, we have $x \in K(x)$ and $F(x)^{T}(y-x) \geq 0 \quad \forall y \in K(x)$. It follows that $x \in \mathbb{C}$ and hence $x \in K(x) \cap \mathbb{C}$. It is easy to see that $F(x)^{T}(y-x) \geq 0$ for every $y \in K(x) \cap \mathbb{C}$. Thus $x$ solves $\mathrm{QVI}(K \cap \mathbb{C}, F)$.

"卫" Let $x \in K(x) \cap \mathbb{C}$ solve $\mathrm{QVI}(K \cap \mathbb{C}, F)$ and let $y \in K(x)$ be arbitrary. By definition in (1), for every $i \in \mathcal{N}$, the point $\left(y_{i}, x^{-i}\right) \in \mathbb{C}$. Furthermore, since $x \in K(x)$, the point $\left(y_{i}, x^{-i}\right)$ also belongs to $K(x)$ for each $i \in \mathcal{N}$. Thus for each $i \in \mathcal{N},\left(y_{i}, x^{-i}\right) \in K(x) \cap \mathbb{C}$. Since $x$ solves $\mathrm{QVI}(K \cap \mathbb{C}$, $F)$, we have

$$
F(x)^{T}\left(\left(y_{i}, x^{-i}\right)-x\right) \geq 0, \quad \text { i.e., } \quad F_{i}(x)^{T}\left(y_{i}-x_{i}\right) \geq 0,
$$


for each $i \in \mathcal{N}$. Summing (2) over all $i \in \mathcal{N}$ we get $F(x)^{T}(y-x) \geq 0$. Since $y$ was an arbitrary point in $K(x), x$ solves $\mathrm{QVI}(K, F)$.

It is easy to see the issue arising in Example 1 does not arise when $L(\cdot) \equiv K(\cdot) \cap \mathbb{C}$. Indeed $L(\cdot)=K(\cdot) \cap \mathbb{C}$ satisfies Harker's conditions, i.e. (i), (ii) from Theorem 4 , with $A=\mathbb{C}$. Thus for shared constraint games Theorem 4 always applies to $\mathrm{QVI}(K \cap \mathbb{C}, F)$. And when Theorem 4 is applied with $L(\cdot)=K(\cdot) \cap \mathbb{C}$, one obtains immediately Theorem 7 of Facchinei et al..

Theorem 9 Let $F$ be continuous and $K$ be as defined by (1). Then every solution of $V I(\mathbb{C}, F)$ is a solution of $Q V I(K, F)$.

Proof : In Theorem 4 , take $L(\cdot):=K(\cdot) \cap \mathbb{C}$. It is not hard to see that $A=\mathbb{C}$ satisfies conditions Theorem 4(i),(ii) with this $L$ :

(i) $K(x) \cap \mathbb{C} \subseteq \mathbb{C}$ for all $x \in \mathbb{C}$

(ii) $x \in K(x) \cap \mathbb{C}$ for all $x \in \mathbb{C}$ (cf. Lemma 3(b))

Now applying Theorem 4 gives

$$
S O L(V I(\mathbb{C}, F))=S O L(V I(A, F)) \subseteq S O L(Q V I(L, F))=S O L(Q V I(K \cap \mathbb{C}, F)) .
$$

But by Proposition 8, SOL $(Q V I(K \cap \mathbb{C}, F))=S O L(Q V I(K, F))$. Consequently

$$
S O L(V I(\mathbb{C}, F)) \subseteq S O L(Q V I(K, F)),
$$

which is the result of Theorem 7 .

In other words Harker's approach, with an application to $\mathrm{QVI}(K \cap \mathbb{C}, F)$, in fact subsumes the approach of Facchinei et al.. The interested reader may also check that Theorem 5 applies even with ' $K(x)$ ' replaced by ' $K(x) \cap \mathbb{C}$ ' in Theorem $5(\mathrm{~b}),(\mathrm{c})$. (The proof requires an exact repetition of the steps used to prove Theorem 5.) Thus for the $\mathrm{QVI}(K \cap \mathbb{C}, F)$ too, the only set with a nonempty interior satisfying Harker's conditions is $\mathbb{C}$ itself.

\section{Concluding remarks}

Equilibria of generalized Nash games are wholly captured by quasi-variational inequalities. However, such objects are analytically less tractable. Accordingly, Harker [9] presented a result which showed that a suitable variational inequality gives a solution to the original QVI. Unfortunately, the direct application of this result to the class of shared-constraint Nash games proves challenging for its hypothesis are hard to satisfy. We examine this issue more carefully and show that under mild assumptions, it is, in fact, impossible to satisfy the hypothesis of Harker's result, when it is applied in a direct manner. In fact, we provide a formal result to support observations made by Facchinei et al. [4]; in particular, they suggested that Harker's result was only applicable to shared-constraint Nash games when $\mathbb{C}$ was rectangular.

We proceed to show that Harker's result can indeed be applied to this class of games, albeit in an indirect manner, by an application to a modified QVI for which the hypothesis are always satisfied. The modified QVI has the same solution set as the original QVI and allows us to independently derive an analogous result of Facchinei et al. [4]. 


\section{A Proof of Lemma 3}

(a) Take any $i \in \mathcal{N}$ and consider an $x \in \mathbb{R}^{m}$. Note from (1) and the cartesian nature assumed on $\mathbb{C}$ that $K_{i}\left(x^{-i}\right)=\left\{y_{i} \in \mathbb{R}^{m_{i}} \mid\left(y_{i}, x^{-i}\right) \in \mathbb{C}\right\}=\left\{y_{i} \in \mathbb{R}^{m_{i}} \mid y_{i} \in \mathbb{C}_{i}, x_{j} \in \mathbb{C}_{j}, j \neq i\right\}$, which is nonempty if $x_{j} \in \mathbb{C}_{j}, \forall j \neq i$. Thus $K(x)=\prod K_{i}\left(x^{-i}\right) \neq \emptyset$ if and only if $x \in \mathbb{C}$. Similarly, for $x \in \mathbb{C}$, we have $y \in K(x)$ if and only if $y \in \mathbb{C}$. Therefore $K(x)=\mathbb{C}$ if and only if $x \in \mathbb{C}$.

(b) Let $x \in K(x)$ implying that $x_{i} \in K_{i}\left(x^{-i}\right), \forall i \in \mathcal{N}$, and therefore $\left(x_{i}, x^{-i}\right) \in \mathbb{C}, \forall i \in \mathcal{N}$ and $x \in \mathbb{C}$. The converse follows by noting that $x \in \mathbb{C}$ is equivalent to $\left(x_{i}, x^{-i}\right) \in \mathbb{C} \forall i$, i.e. $x_{i} \in K_{i}\left(x^{-i}\right), \forall i$ and therefore $x \in K(x)$.

(c) Let $x \in \operatorname{dom}(K)$ and $y, z \in K(x)$, i.e. for each $i \in \mathcal{N},\left(y_{i}, x^{-i}\right)$ and $\left(z_{i}, x^{-i}\right) \in \mathbb{C}$. The convexity of $K(x)$ follows by noting that since $\mathbb{C}$ is convex, $\left(\left(\alpha y_{i}+(1-\alpha) z_{i}\right), x^{-i}\right) \in \mathbb{C}$ for each $i$ and $\alpha \in[0,1]$.

To show closedness, consider a sequence $\left\{y^{k}\right\} \subseteq K(x)$ with limit point $\bar{y}$. By closedness of $\mathbb{C}$, for each $i$, the sequence $\left\{\left(y_{i}^{k}, x^{-i}\right)\right\} \subseteq \mathbb{C}$ and $\lim \left(y_{i}^{k}, x^{-i}\right)=\left(\bar{y}_{i}, x^{-i}\right) \in \mathbb{C}$. Thus $K(x)$ is closed.

(d) Suppose $x \in \operatorname{int}(\mathbb{C})$. Then there exist open sets $\mathcal{O}_{i} \subseteq \mathbb{R}^{m_{i}}$ containing $x_{i}$ such that $x \in \mathcal{O}:=$ $\prod_{i \in \mathcal{N}} \mathcal{O}_{i} \subseteq \mathbb{C}$. Then $\left(\mathcal{O}_{i}, x^{-i}\right):=\cup_{y_{i} \in \mathcal{O}_{i}}\left(y_{i}, x^{-i}\right) \subseteq \mathbb{C}$, so that $\mathcal{O}_{i} \subseteq K_{i}\left(x^{-i}\right)$, for each $i \in \mathcal{N}$. It follows that $\mathcal{O} \subseteq K(x)$.

For the converse, let $\operatorname{int}(K(x))$ be nonempty and $x \in \operatorname{int}(K(x))$. Then for each $i \in \mathcal{N}, x_{i}$ belongs the interior of $K_{i}\left(x^{-i}\right)$ (where $K_{i}\left(x^{-i}\right)$ is considered a set in $\mathbb{R}^{m_{i}}$ ). Thus there exist open sets $\mathbb{R}^{m_{i}} \supseteq \mathcal{O}_{i} \subseteq K_{i}\left(x^{-i}\right)$ containing $x_{i}$ for all $i$. It follows that $\left(\mathcal{O}_{i}, x^{-i}\right) \subseteq \mathbb{C}$ for all $i \in \mathcal{N}$. Now since $\mathbb{C}$ is convex, the average of these sets is contained in $\mathbb{C}$, i.e.

$$
\mathcal{A}:=\sum_{i \in \mathcal{N}} \frac{\left(\mathcal{O}_{i}, x^{-i}\right)}{N}=\frac{1}{N} \prod_{i \in \mathcal{N}} \mathcal{O}_{i}+\frac{N-1}{N} x \subseteq \mathbb{C} .
$$

Since $x_{i} \in \mathcal{O}_{i}, \mathcal{A}$ contains $x$. Furthermore, $\mathcal{A}$ is open, implying that $x \in \operatorname{int}(\mathbb{C})$.

\section{References}

[1] K. Arrow and G. Debreu. Existence of an equilibrium for a competitive economy. Econometrica, 22(3):290, 265, 1954.

[2] A. Bensoussan, M. Goursat, and J.L. Lions. Contrôle impulsionnel et inequations quasivariationnelles stationnaires. Comptes Rendus des Séances de l'Académie des Sciences, 276:1279$1284,1973$.

[3] D. Chan and J. S. Pang. The generalized Quasi-Variational inequality problem. Mathematics of Operations Research, 7(2):211-222, May 1982.

[4] F. Facchinei, A. Fischer, and V. Piccialli. On generalized Nash games and variational inequalities. Operations Research Letters, 35(2):159-164, 2007.

[5] F. Facchinei and C. Kanzow. Generalized Nash equilibrium problems. 4OR: A Quarterly Journal of Operations Research, 5(3):173-210, 2007.

[6] F. Facchinei and J-S. Pang. Finite-Dimensional Variational Inequalities and Complementarity Problems I. Springer, 1 edition, February 2003. 
[7] I. Fonseca and W. Gangbo. Degree Theory in Analysis and Applications. Clarendon Press, April 1995.

[8] A. M. Glicksman. An Introduction to Linear Programming and the Theory of Games. Courier Dover Publications, 2001.

[9] P. T. Harker. Generalized Nash games and quasi-variational inequalities. European Journal of Operational Research, 54(1):81-94, September 1991.

[10] T. Ichiishi. Game theory for economic analysis. Academic Press, 1983.

[11] A. A. Kulkarni and U. V. Shanbhag. On the variational equilibrium as a refinement of the generalized Nash equilibrium. Under review with Automatica, 2009.

[12] S. Kumaresan. Topology of metric spaces. Alpha Science Int'l Ltd., 2005.

[13] R. Rockafellar. Convex analysis. Princeton University Press, Princeton, NJ, 1997. Reprint of the 1970 original, Princeton Paperbacks.

[14] J. B. Rosen. Existence and uniqueness of equilibrium points for concave n-person games. Econometrica, 33(3):520-534, July 1965. 